\title{
CRT-D Upgrading in a Patient with Persistent Left Superior Vena Cava and Right Superior Vena Cava Atresia Using the Novel Active-Fixation Quadripolar Left Ventricular Lead
}

\author{
Sergio Conti ${ }^{1,2}$, Antonio Taormina ${ }^{1,3}$, Vito Bonomo ${ }^{1,4}$, Umberto Giordano $^{1}$, Giuseppe Sgarito ${ }^{1}$ \\ ${ }^{1}$ ARNAS Ospedale Civico Di Cristina Benfratelli, Palermo, Italy \\ ${ }^{2}$ University of Tor Vergata, Rome, Italy \\ ${ }^{3}$ University of Messina, Messina, Italy \\ ${ }^{4}$ University of Palermo, Palermo, Italy \\ Email: *sergioconti.md@gmail.com
}

How to cite this paper: Conti, S., Taormina, A., Bonomo, V., Giordano, U. and Sgarito, G. (2018) CRT-D Upgrading in a Patient with Persistent Left Superior Vena Cava and Right Superior Vena Cava Atresia Using the Novel Active-Fixation Quadripolar Left Ventricular Lead. World Journal of Cardiovascular Diseases, 8, 462-466. https://doi.org/10.4236/wjcd.2018.89045

Received: April 24, 2018

Accepted: September 24, 2018

Published: September 27, 2018

Copyright $\odot 2018$ by authors and Scientific Research Publishing Inc. This work is licensed under the Creative Commons Attribution International License (CC BY 4.0).

http://creativecommons.org/licenses/by/4.0/

\begin{abstract}
The persistence of left superior vena cava (PLSVC) is the most common congenital anomaly of the venous return system to the heart. Because of the increasing number of patients referred for cardiac resynchronization therapy (CRT) devices implantations, it is expected to encounter this venous anatomic variation. Left ventricular lead placement at an appropriate site is an integral and technically challenging part of successful CRT. In case of cardiac abnormalities could be difficult to achieve an optimal cardiac rhythm management devices implantation. Previous reports in patients with PLSVC highlighted the challenges to achieve an optimal cardiac rhythm device implantation. Recently, a new quadripolar active fixation left ventricular lead is available for CRT device implantation. Hereby we report a case of a device upgrading from dual-chamber pacemaker to CRT with defibrillator backup using the active fixation left ventricular quadripolar lead in a patient with PLSVC and right superior vena cava atresia.
\end{abstract}

\section{Keywords}

Cardiac Resynchronization Therapy, Anatomic Abnormalities, Persistent Left Superior Vena Cava, Right Vena Cava Atresia, Active-Fixation Lead

\section{Introduction}

The persistence of left superior vena cava (PLSVC) is the most common conge- 
nital anomaly of the venous return system to the heart. Because of the increasing number of patients referred for cardiac resynchronization therapy (CRT) devices implantations, it is expected to encounter this venous anatomic variation. Left ventricular lead placement at an appropriate site is an integral and technically challenging part of successful CRT. The final position of the lead depends on the anatomy of the cardiac veins, the stability of the lead, and the quality of pacing parameters. Previous reports of cardiac rhythm device implantation in patients with PLSVC highlighted the challenges to achieve a stable of the leads inside the heart chambers. Improvements in materials and techniques over the years allowed the successful implantation of cardiac rhythm management devices including CRT devices even in case of unfavorable anatomies. Recently, a new quadripolar active fixation left ventricular lead has been developed and available for CRT device implantation. This new technology combines the advantages of the multipolar technology and the stability of the active fixation leads and it allows a more precise and stable lead placement, particularly helpful in case of abnormal or unfavorable anatomy of the coronary sinus.

\section{Case Report}

A 77-year-old man suffering from congestive heart failure (NYHA class II) and post-ischemic dilated cardiomyopathy was referred to our Centre for an upgrading to a cardiac resynchronization therapy device with defibrillator backup (CRT-D). Patient had a dual-chamber pacemaker (PM) implantation due to symptomatic bradycardia 7 years before. During PM implantation, a persistent left superior vena cava (PLSVC) with right superior vena cava (SVC) atresia was documented. For this reason, a dual-chamber PM was implanted using the left-side approach and the leads were advanced through the PLSVC and coronary sinus (CS) and placed into the right atrial appendage and the right ventricular apex. Current baseline ECG revealed sinus rhythm, left bundle branch clock with a QRS complex duration of $180 \mathrm{~ms}$. Transthoracic echocardiography showed a dilated left ventricle and a left ventricular ejection fraction of $30 \%$.

The procedure was performed in fasting state and in the usual sterile fashion. Cannulation of the left subclavian vein was performed. Venography of the left subclavian vein confirmed patency of the left subclavian vein and showed PLSVC draining into a hugely dilated CS. We decide to explore the CS with a standard $6 \mathrm{~F}$ pig-tail catheter and contrast injections pulling back the diagnostic catheter from the CS ostium to the PLSVC. At first, there was no evidence of vein branches taking off from the main body of the CS. We decided to explore the CS drawing back and rotating a subselector catheter (Attain Select ${ }^{\mathrm{TM}} \mathrm{II}$, Medtronic, MN, USA). A small and sharp-angle vein was selectively cannulated with an inferior-to-superior direction (Figure 1(a)). However, this posterior branch was too tortuous and with a sharp takeoff to let the wire advance deep into the vein. We found a less tortuous and angled posterolateral vein and we were able to advance the wire deep into it (Figure 1(b)). A quadripolar 


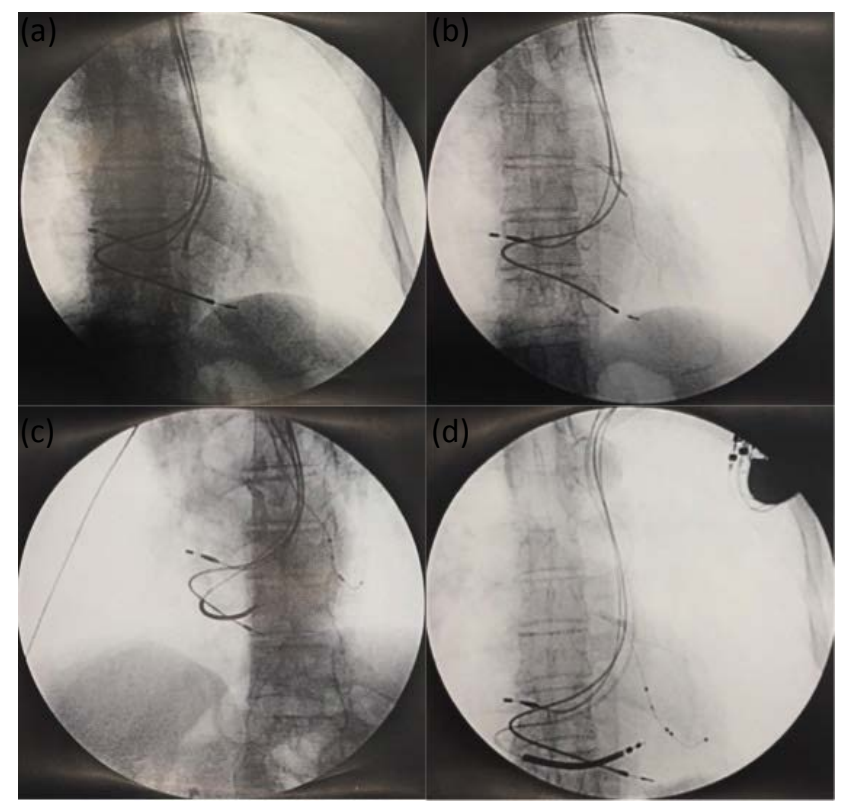

Figure 1. (a) Antero-posterior view: the sub-selector was selectively engaging a small and sharp angulate branch with an inferior-to-superior direction. It is also shown the previously implanted pacemaker leads; (b) Antero-posterior view: the sub-selector was retracted and a posterolateral vein was engaged with a standard wire; (c) Left anterior oblique view: the quadripolar active-fixation catheter was advanced into the posterolateral vein. It is also shown the new single-coil active fixation defibrillator lead implanted in the mid-septal region; (d) Antero-posterior view: final position of the leads.

active-fixation (Attain Stability ${ }^{\mathrm{TM}}$, Medtronic, MN, USA) was advanced into the posterolateral vein (Figure 1(c) and Figure 1(d)). The Attain Stability ${ }^{\mathrm{TM}}$ was implanted as recommended by manufacturer [1]. Briefly, the lead was advance to the desired position, where pacing parameters were satisfactory and phrenic nerve stimulation absent. The helix was then positioned against the vessel and the lead was rotated clockwise until resistance was felt. Stability was tested by a pull and push test. Finally, parameters were checked again. Stimulation threshold was $0.9 \mathrm{~V}$ at $0.5 \mathrm{~ms}$, impedance $1119 \mathrm{Ohms}$, R wave amplitude $14.1 \mathrm{mV}$. Once the left ventricular lead was implanted, a single-coil active fixation defibrillator lead was positioned into the mid-septal region of the right ventricle. The previously implanted atrial lead was tested to confirm the good functional status of the lead and connected to the CRT-D device. The patient was discharged uneventfully three days after the procedure and the first device follow-up at one month confirmed satisfactory electrical parameters.

\section{Discussion}

The persistence of left superior vena cava (LSVC) is the most common congenital anomaly of the venous return system to the heart. PLSVC can be isolated or associated with other congenital heart diseases and it has been reported in about $0.5 \%$ of the general population and up to $10 \%$ in patients with congenital heart disease [2]. Usually, it is totally asymptomatic, and can be generally encountered 
as an incidental finding during cardiac investigations (i.e. echocardiography, computed tomography) or during any intervention involving catheterization of the right cardiac cavities, such as anti-bradycardia or anti-tachycardia devices implantation [3].

Briefly, development of the SVC system begins with the sinus venosus, in which 3 pairs of veins (cardinal, umbilical, and vitelline) drain. The right cardinal vein is transformed into right SVC, the left cardinal vein into LSVC, and the left part of the sinus venosus into the coronary sinus (CS). During the normal fetal development, there is progressive involution and disappearance of the left-sided anterior venous cardinal system, leaving the CS and the ligament of Marshall. Failure of the closure of the left anterior cardinal vein results in PLSVC [4]. This anatomical variant has been associated with an increased lifetime risk of developing atrial fibrillation [5]. In patients with persistence of LSVC, RSVC is frequently present. However, isolated PLSVC with an absent right SVC is rarely reported with an incidence of $0.07 \%-0.13 \%$ [6]. Published reports of cardiac rhythm management devices highlight the challenges to place stable leads inside the right chambers. Use of preformed loop stylet and active fixation leads has enabled the operator to successfully place stable pacing and defibrillator leads. Nowadays, because of increasing patients referred for CRT devices implants, it is expected to encounter this venous anatomic variation and in particular to find major challenges in placing the left ventricular pacing lead in the CS branches. Catheterization of these branches may be particularly challenging, because of the sharp take off angle of the veins from the CS main body. Moreover, the presence of severe dilatation of the CS precludes adequate support for delivery system. Balloon occlusion retrograde angiography may be not possible because of the size of the CS.

Recently, a new quadripolar active fixation left ventricular lead has been developed and available for CRT device implantation. Combining together the advantages of the multipolar technology and the stability of the active fixation leads, this new technology adds an important tool in the portfolio of the electrophysiology operators. It allows a more precise lead placement and stability, particularly helpful in case of abnormal or unfavorable anatomies of the CS. To the best of our knowledge, this is the first report of Attain Stability ${ }^{\mathrm{TM}}$ implantation in a patient with a PLSVC and right SVC atresia. The Attain Stability ${ }^{\mathrm{TM}}$ left ventricular lead is an innovative active fixation lead with a helix attached before the proximal electrode to help fix the lead in any desired position. The lead could be easily maneuvered and it could be repositioned at the desired location if required. This lead has the potential to be used in all types of CS anatomies.

\section{Conclusion}

Left ventricular lead placement at an appropriate site is an integral and technically challenging part of successful CRT. The final position of the lead depends on the anatomy of the cardiac veins and on the stability of the lead. Challenging 
anatomical variants, often discovered at the time of the procedure, can pose difficulties and complications during central venous cannulation or device implantation such as arrhythmia, cardiogenic shock, cardiac tamponade, CS dissection and thrombosis. However, improvements in materials and techniques over the years allowed the successful implantation of cardiac rhythm management devices including CRT devices. Although the procedure was technically challenging, currently available technologies enabled the implantation of CRT devices by a totally transvenous approach even in patients with PLSVC and right SVC atresia.

\section{Conflicts of Interest}

The authors declare no conflicts of interest regarding the publication of this paper.

\section{References}

[1] Yee, R., Gadler, F., Hussin, A., Omar, R.B., Khaykin, Y., Verma, A., Lazeroms, M., Hine, D.S. and Marquard, K.R. (2014) Novel Active Fixation Mechanism Permits Precise Placement with a Left Ventricular Lead: Early Results from a Multicenter Clinical Study. Heart Rhythm, 11, 1150-1155. https://doi.org/10.1016/j.hrthm.2014.04.020

[2] Irwin, R.B., Greaves, M. and Schmitt, M. (2012) Left Superior Vena Cava: Revisited. European Heart Journal, 13, 284-291.

[3] Biffi, M., Boriani, G., Frabetti, L., Bronzetti, G. and Branzi, A. (2001) Left Superior Vena Cava Persistence in Patients Undergoing Pacemaker or Cardioverter-Defibrillator Implantation: A 10-Year Experience. Chest, 120, 139-144. https://doi.org/10.1378/chest.120.1.139

[4] Mora, G. (2014) A Novel Method of Placing Right Ventricular Leads in Patients with Persistent Left Superior Vena Cava Using a Conventional J Stylet. Indian Pacing and Electrophysiology Journal, 14, 65-74. https://doi.org/10.1016/S0972-6292(16)30731-8

[5] Hsu, L.F., Jaïs, P., Keane, D., Wharton, J.M., Deisenhofer, I., Hocini, M., Shah, D.C., Sanders, P., Scavée, C., Weerasooriya, R., Clémenty, J. and Haïssaguerre, M. (2004) Atrial Fibrillation Originating From Persistent Left Superior Vena Cava. Circulation, 109, 828-832. https://doi.org/10.1161/01.CIR.0000116753.56467.BC

[6] Cheng, Z., Deng, H., Cheng, K., Chen, T., Gao, P. and Fang, Q. (2013) Implantation of a Pacemaker in a Patient with Persistent Left Superior Vena Cava and Absence of Right Superior Vena Cava. International Journal of Cardiology, 168, e53-e54. https://doi.org/10.1016/j.ijcard.2013.06.108 